\title{
THE FIRST DERIVATIVE OF THE PERIOD FUNCTION OF A PLANE VECTOR FIELD
}

\author{
J.-P. Françoise
}

Abstract

The algorithm of the successive derivatives introduced in [5] was implemented in [7], [8]. This algorithm is based on the existence of a decomposition of 1 -forms associated to the relative cohomology of the Hamiltonian function which is perturbed. We explain here how the first step of this algorithm gives also the first derivative of the period function. This includes, for instance, new presentations of formulas obtained by Carmen Chicone and Marc Jacobs in [3].

\section{Introduction}

Let $X_{\epsilon}$ be a vector field of the type:

$$
X=x \frac{\partial}{\partial y}-y \frac{\partial}{\partial x}+\epsilon \sum_{2 \leq i+j \leq n} a_{i j} x^{i} y^{j} \frac{\partial}{\partial x}+b_{i j} x^{i} y^{j} \frac{\partial}{\partial y} .
$$

$\left(\left(a_{i j}, b_{i j}\right) \in \mathbb{R}^{2}\right), \epsilon$ is a parameter.

For a vector field $X_{\epsilon}$ corresponding to fixed values of $\left(a_{i j}, b_{i j}\right)$, there is a neighborhood $U$ of the origin $0 \in \mathbb{R}^{2}$ on which the flow of $X_{\epsilon}$, solution of the differential system:

$$
\left\{\begin{array}{l}
\dot{x}=-y+\epsilon \sum_{2 \leq i+j \leq n} a_{i j} x^{i} y^{j} \\
\dot{y}=x+\epsilon \sum_{2 \leq i+j \leq n} b_{i j} x^{i} y^{j}
\end{array}\right.
$$

exists for all initial values which belong to this neighborhood.

There is furthermore a first return mapping $L_{\epsilon}$ defined on $U$. Given for instance, an initial point $(r, 0), r>0$ the solution of equation (2) 
with initial data $(r, 0)$ intersects again for the first time the $x$-axis at some point $\left(L_{\epsilon}(r), 0\right), L_{\epsilon}(r)>0$. We denote by $\Sigma=\{(x, 0) \in U\}$ the transversal section. By transversality, the mapping $L_{\epsilon}$ is analytic and it can be represented as a convergent Taylor series:

$$
L_{\epsilon}(r)=r+L_{2}(r) \epsilon^{2}+\cdots+L_{k}(r) \epsilon^{k}+\cdots
$$

In [5], an algorithm was introduced to compute the first non-vanishing coefficient $L_{k 0}(r)(\underline{a}, \underline{b})$ for a homogeneous perturbative part. It was later implemented in [7], [8].

Introduce now $\gamma_{\epsilon}$ the arc of the trajectory of $X_{\epsilon}$ between the two points $(r, 0)$ and $\left(L_{\epsilon}(r), 0\right)$ and $T_{\epsilon}$ the time along the flow between these two points. We give here a practical formula to compute the first derivative $\left.\frac{\partial T_{\epsilon}}{\partial \epsilon}\right|_{\epsilon=0}$. This formula is based on the relative cohomology decomposition of 1 -forms used in preceding articles [5], [7], [8].

\section{The relative cohomology decomposition of 1-forms}

We use complex coordinates $z=(1 / \sqrt{2})(x+i y), \bar{z}=(1 / \sqrt{2})(x-i y)$; and the 1-form $\omega_{\epsilon}=i_{X_{\epsilon}}(d x \wedge d y)$. With these new notations, we use:

$$
\omega_{\epsilon}=d H+\epsilon \omega_{1}=d H+\epsilon \sum_{2 \leq i+j \leq d} A_{i j} z^{i} \bar{z}^{j} d z+\bar{A}_{i j} \bar{z}^{i} z^{j} d \bar{z} .
$$

The complex coefficients $A_{i j}$ of equation (4) are easily related to the real coefficients $\left(a_{i j}, b_{i j}\right)$. The function $H$ is $H:(z, \bar{z}) \mapsto H(z, \bar{z})=z \bar{z}$. We remind without proof the following

Proposition 1. Any polynomial 1-form $\omega$ can be decomposed into

$$
\omega=g d H+d R+(1 / 2) \psi(H)[z d \bar{z}-\bar{z} d z]
$$

where $g, R$ are polynomials $(R(0)=0)$ in $(z, \bar{z})$ and $\psi$ is a polynomial in one variable.

To compute $g$ and $\psi(H)$, take $d \omega=F(z, \bar{z})$. Write

$$
F(z, \bar{z})=\sum_{i \neq j} F_{i j} z^{i} \bar{z}^{j}+\sum_{i=j} F_{i j}(z \bar{z})^{i}
$$

then

$$
g=\sum_{i \neq j} \frac{F_{i j} z^{i} \bar{z}^{j}}{i-j}
$$


To find $\psi$, take

$$
\phi(t)=\sum_{i} F_{i i} t^{i} .
$$

Then solve $t \psi^{\prime}(t)+\psi(t)=\phi(t)$. Now get $R$ by applying Poincaré's theorem to

$$
d \omega-d g \wedge d H-\phi(H) d z \wedge d \bar{z} .
$$

In the sequel, we describe the method given in [5] to get the successive derivatives of the return mapping.

A classical formula gives:

$$
L_{1}\left(r, A_{i j}, \bar{A}_{i j}\right)=-\int_{H=r} \omega_{1} .
$$

Assume that $L_{1}\left(r, A_{i j}, \bar{A}_{i j}\right) \equiv 0$ (as a function of $r$ ) then there is a polynomial $g_{1}$ such that

$$
\omega_{1}=g_{1} d H+d R_{1}
$$

And we get

$$
L_{2}\left(r, A_{i j}, \bar{A}_{i j}\right)=-\int_{H=r} g_{1} \omega_{1} .
$$

One can show inductively that given

$$
L_{k}\left(r, A_{i j}, \bar{A}_{i j}\right)=-\int_{H=r} g_{k-1} \omega_{1},
$$

if $L_{k}\left(r, A_{i j}, \bar{A}_{i j}\right) \equiv 0$ (as a function of $r$ ), then there is a polynomial $g_{k}$ such that

$$
g_{k-1} \omega_{1}=g_{k} d H+d R_{k}
$$

and then

$$
L_{k+1}\left(r, A_{i j}, \bar{A}_{i j}\right)=-\int_{H=r} g_{k} \omega_{1} .
$$

As a consequence, we can compute the first non-zero coefficient $L_{k}\left(r, A_{i j}, \bar{A}_{i j}\right)$ by building the sequence of polynomials $g_{1}, \ldots, g_{k}, \ldots$

At each step $k$ we have a 1 -form $g_{k} \omega_{1}$. We must first compute the differential $d\left(g_{k} \omega_{1}\right)=F^{k}(z, \bar{z}) d z \wedge d \bar{z}$. We then split into two parts:

$$
F^{k}(z, \bar{z})=\sum_{i \neq j} F_{i j}^{k} z^{i} \bar{z}^{j}+\sum_{i=j} F_{i j}^{k}(z \bar{z})^{i} .
$$


We find that

$$
L_{k+1}\left(r, A_{i j}, \bar{A}_{i j}\right)=\sum F_{l l}^{k} r^{l}
$$

and we introduce

$$
g_{k+1}=\sum_{i \neq j} \frac{F_{i j}^{k} z^{i} \bar{z}^{j}}{i-j}
$$

Next we compute $d\left(g_{k+1} \omega_{1}\right)$ and we repeat the process.

\section{The first derivative of the period}

We write $T_{\epsilon}=T_{0}+\epsilon T_{1}+O\left(\epsilon^{2}\right)\left(T_{0}=2 \pi\right)$. Let $\varpi_{0}=d \theta$ then $\varpi_{0} \wedge d H=$ $r d \theta \wedge d r=d x \wedge d y$ and $\int_{\gamma_{\epsilon}} \varpi_{0}=2 \pi=T_{O}$.

Definition 2. A 1-form $\varpi_{0}+\epsilon \varpi_{1}+\cdots+\epsilon^{k} \varpi_{k}$ is said to be $k$-isochronous to $X_{\epsilon}$ if $\left(\varpi_{0}+\epsilon \varpi_{1}+\cdots+\epsilon^{k} \varpi_{k}\right)\left(X_{\epsilon}\right)=1+O\left(\epsilon^{k+1}\right)$.

Proposition 3. Let $\varpi_{0}+\epsilon \varpi_{1}$ be a 1-isochronous 1-form, then $T_{\epsilon}=\int_{\gamma_{\epsilon}}\left(\varpi_{0}+\epsilon \varpi_{1}\right)+O\left(\epsilon^{2}\right)=2 \pi+\epsilon \int_{H=r^{2}} \varpi_{1}+O\left(\epsilon^{2}\right)$.

Proof:

$\int_{\gamma_{\epsilon}}\left(\varpi_{0}+\epsilon \varpi_{1}\right)=\int_{\gamma_{\epsilon}}\left(\varpi_{0}+\epsilon \varpi_{1}\right)\left(X_{\epsilon}\right) d t=\int_{\gamma_{\epsilon}} d t+O\left(\epsilon^{2}\right)=T_{\epsilon}+O\left(\epsilon^{2}\right)$.

We now try to build a 1-isochronous form. This yields:

$$
\left(\varpi_{0}+\epsilon \varpi_{1}\right) \wedge\left(d H+\epsilon \omega_{1}\right)=\left(1+O\left(\epsilon^{2}\right)\right) d x \wedge d y
$$

The 1-form $\varpi_{1}$ should be such that

$$
\varpi_{1} \wedge d H=-\varpi_{0} \wedge \omega_{1}
$$

Now the relative cohomology decomposition of $\omega_{1}$ displays:

$$
\omega_{1}=g d H+d R+(1 / 2) \psi(H)[z d \bar{z}-\bar{z} d z] .
$$

This yields to a possible choice for $\varpi_{1}$ :

$$
\varpi_{1}=-\left[g+(1 / r)\left(\frac{\partial R}{\partial r}\right)\right] \varpi_{0}
$$


Now, we obtain with this choice of $\varpi_{1}$ :

$$
T_{1}=\int_{H=r^{2}} \varpi_{1}=-\int_{H=r^{2}}\left[g+(1 / r)\left(\frac{\partial R}{\partial r}\right)\right] d \theta .
$$

Now, we remark that the construction of $g$ yields: $\int_{H=r^{2}} g d \theta=0$.

We expand $R$ :

$$
R(z, \bar{z})=\sum_{i \neq j} R_{i j} z^{i} \bar{z}^{j}+\sum_{i=j} R_{i i}(z \bar{z})^{i}
$$

and get ultimately:

$$
T_{1}(r)=-4 \pi \sum_{k} k R_{k k} r^{(2 k-2)}
$$

To summarize, the first derivative $\left.\frac{\partial T_{\epsilon}}{\partial \epsilon}\right|_{\epsilon=0}$ is essentially given by the part in the Taylor development of $R$ which depends only in $H=z \bar{z}$. An equivalent expression was already derived by C. Chicone and M. Jacobs in $[3$, Lemma 3.2 , p. 455]. Our main point here was to clear up the link of their result with the relative cohomology of forms.

\section{The case of an homogeneous perturbative part}

To illustrate the preceding formula, we consider the situation where the perturbative part $\omega_{1}$ is homogeneous of degree $n$. So we consider now the 1 -form

$$
\omega_{\epsilon}=d H+\epsilon \omega_{1}=d H+\epsilon \sum_{2 \leq i+j=n} A_{i j} z^{i} \bar{z}^{j} d z+\bar{A}_{i j} \bar{z}^{i} z^{j} d \bar{z}
$$

We first compute $d \omega_{1}=D(z, \bar{z}) d z \wedge d \bar{z}$

$$
D(z, \bar{z})=\sum_{i+j=n}\left[-j A_{i j} z^{i} \bar{z}^{j-1}+j \bar{A}_{i j} \bar{z}^{i} z^{j-1}\right]
$$


There is a contribution to $\phi(H)$ only if $i=j-1$, hence $n+1=2 j$. So $n$ must be odd. Write $n=2 J-1$, then $i=J-1$ and

$$
\phi(H)=-2 J \operatorname{Im}\left(A_{J-1 J}\right) H^{(J-1)} .
$$

So the first condition for a center is $\operatorname{Im}\left(A_{J-1 J}\right)=0[\mathbf{5}]$. We have now:

$$
g=\sum_{i \neq j-1}\left[-j A_{i j} z^{i} \bar{z}^{j-1}+j \bar{A}_{i j} \bar{z}^{i} z^{j-1}\right] /(i-j+1) .
$$

Note that $g$ is homogeneous of degree $n-1$. We have then to consider

$$
\omega_{1}-g d H-(1 / 2) \psi(H)[z d \bar{z}-\bar{z} d z]=\Omega
$$

Note that $\Omega$ is homogeneous of degree $n+1$, then we obtain $\Omega=$ $d\left[\left(i_{C} \Omega /(n+1)\right]\right.$ where $C=z \frac{\partial}{\partial z}+\bar{z} \frac{\partial}{\partial \bar{z}}$ is the Euler vector field. So we can choose

$$
R=\left[\omega_{1}(C)-2 g H\right] /(n+1) .
$$

Now we have to isolate the monomials in $R$ for which the exponent of $z$ equals the exponent of $\bar{z}$. There are no such monomials in $g H$. Hence it is only necessary to look at those in $\omega_{1}(C)$. This yields

$$
\omega_{1}(C)=\sum_{i+j=n}\left[A_{i j} z^{i+1} \bar{z}^{j}+\bar{A}_{i j} \bar{z}^{i+1} z^{j}\right] .
$$

The monomials in $\omega_{1}(C)$ such that $i+1=j$ and $i+j=n$ exist only if $n=2 I+1$ and we ultimately obtain

$$
T_{1}(r)=4 \pi \operatorname{Re}\left(A_{I I+1}\right) r^{2} I .
$$

In particular, this yields the "second" condition for a center to be isochronous

$$
\operatorname{Re}\left(A_{I I+1}\right)=0
$$

if $n=2 I+1$. This condition was previously obtained by $[\mathbf{9}]$. In this article, the authors use the Cherkas transform and deal with the Abel Equation. 


\section{Final remarks on the isochronous centres}

The search for isochronous centres is certainly one the priority subjects in the bifurcation theory of differential systems. To name few of the many important contributions to this field, we mention the articles of $[\mathbf{1}],[\mathbf{2}]$, [10]. Recently, C. J. Christopher and J. Devlin [4] and independently B. Schuman $[\mathbf{1 1}$ proved that the origin is never isochronous in non zero homogeneous hamiltonian perturbations. It may be of some interest to see appropriate extensions to several variables in the general setting provided by normal form theory. (cf. [6]).

\section{References}

1. R. Conti, Centers of quadratic systems, Ricerche Mat. Suppl. 36 (1987), 117-126.

2. R. Conti, Uniformly isochronous centres of polynomial systems, in "Lecture Notes in Pure and Applied Math.," 152, 1994, pp. 21-31.

3. C. Chicone And M. Jacobs., Bifurcations of critical periods for plane vector fields, Trans. Amer. Math. Soc. 312 (1989), 433-486.

4. C. J. Christopher And J. Devlin, Isochronous centers in planar polynomial systems, SIAM J. Math. Anal., (to appear).

5. J.-P. FranÇOISE, Successive derivatives of a first return map, application to quadratic vector fields, Ergodic Theory Dynam. Systems 16 (1996), 87-96.

6. J.-P. FrAnÇOISE, Birkhoff normal forms and analytic geometry, Differential geometry and mathematical physics, Banach Center Publications, Warsaw (1996).

7. J.-P. Françoise And R. Pons, Computer algebra methods and the stability of differential systems, Random Comput. Dynam. 3(4) (1995), 265-287.

8. J.-P. FrançOISE Et R. Pons, Une approche algorithmique du problème du centre pour des perturbations homogènes, Bull. Sci. Math. 120 (1996), 1-17.

9. A. Gasull, A. Guillamon and V. Mañosa, Centre and isochronicity conditions for systems with homogeneous non linearities, Proceedings of the 2nd Catalan days of Applied Mathematics, Font-Romeu, Odeillo (M. Sofonea and J.N. Corvellec, eds.), Presses Universitaires de Perpignan, 1995, pp. 105-116.

10. W. S. Loud, Behavior of the period of solutions of certain plane autonomous systems near centers, Contrib. Differential Equations 3 (1964), 21-36. 
11. B. Schuman, Sur la forme normale de Birkhoff et les centres isochrones, C. R. Acad. Sci. Paris Sér. I Math. 322 (1996), 21-24.

\author{
Institut de Mathematiques \\ Université Pierre et Marie Curie (Paris VI) \\ UFR 920 BP 172 \\ 4 , Place Jussieu \\ 75252 Paris Cedex 05 \\ FRANCE \\ e-mail: jpf@ccr.jussieu.fr
}

Primera versió rebuda el 30 de Novembre de 1996, darrera versió rebuda el 5 de Febrer de 1997 